\title{
LYAR promotes the proliferation of non-small cell lung cancer and is associated with poor prognosis
}

\author{
Xiao-Ning Lu',\#, Guan-Jun Ju ,\#, Yu-Xin Wang ${ }^{3}$, Yong-Liang Wang', Kun Wang1, \\ Jian-Le Chen ${ }^{4}$, Wei Cai ${ }^{5}$, Qi-Wei Zang, **
}

${ }^{1}$ Department of Cardiothoracic Surgery, The Affiliated Suqian First People's Hospital of Nanjing Medical University, Suqian, 223800, Jiangsu, China

${ }^{2}$ Department of Thoracic Surgery, Nantong Tumor Hospital, Nantong, 226001, Jiangsu, China

${ }^{3}$ Department of Gastroenterology, The Affiliated Suqian First People's Hospital of Nanjing Medical University, Suqian, 223800, Jiangsu, China

${ }^{4}$ Department of Thoracic Surgery, Affiliated Hospital of Nantong University, Nantong, 226001, Jiangsu, China

${ }^{5}$ Department of Neurosurgery, The Affiliated Suqian First People's Hospital of Nanjing Medical University, Suqian, 223800, Jiangsu, China

\#These authors contributed equally to the manuscript and thus share the first authorship.

\begin{abstract}
Introduction. The aim of the study was to investigate the clinical significance of Ly-1 antibody reactive clone (LYAR) in non-small-cell lung cancer (NSCLC).

Material and methods. The expressions of LYAR at the protein level in representative paired NSCLC tumor tissues and adjacent non-tumor tissues were measured by Western blot and immunohistochemistry. Kaplan-Meier method was used to calculate the survival curve of patients with NSCLC. Cell Counting Kit-8 assay and flow cytometry were used to estimate the cell proliferation and cell cycle, respectively. Terminal-deoxynucleotidyl-transferase-mediated dUTP-biotin nick end labeling (TUNEL) assay was performed to detect cell apoptosis. Results. LYAR was dramatically overexpressed in NSCLC tissues which were closely related to the survival of patients with NSCLC. In clinical studies, the expression of LYAR was related to the clinical stage, histological differentiation, and Ki-67 expression. A positive correlation was found between LYAR and Ki-67 expression by Spearman's correlation test. After serum starvation for $72 \mathrm{~h}$, serum re-addition significantly increased the expression of LYAR, PCNA, and Cyclin A and promoted the cell cycle progression. LYAR knockdown inhibited the proliferation and induced the G0/G1 cell cycle arrest and apoptosis of A549 cells.

Conclusions. The present study revealed the clinical significance of LYAR in NSCLC. LYAR might serve as a tumor promoter in NSCLC progression by promoting the proliferation and inhibiting the apoptosis of NSCLC cells. Inhibiting the expression of LYAR was considered as a potential novel therapeutic strategy for NSCLC. (Folia Histochemica et Cytobiologica 2021, Vol. 59, No. 4, 282-290)
\end{abstract}

Key words: non-small-cell lung cancer; Ly-1 antibody reactive clone; A549 cells; cell cycle; prognosis

*Correspondence address: Qi-Wei Zang Department of Cardiothoracic Surgery, The Affiliated Suqian First People's Hospital of Nanjing Medical University, No.120, Suzhi Road, Suqian, 223800, Jiangsu, China phone: +8618012186865 e-mail: sqzangqiwei@163.com

\author{
Co-authors e-mail: \\ Xiao-Ning Lu, 409669002@qq.com; Guan-Jun Ju, \\ juguanjun540@163.com; \\ Yu-Xin Wang, wangyuxin87@126.com; \\ Yong-Liang Wang, 308342806@qq.com; \\ Kun Wang, 15862253671@163.com; \\ Jian-Le Chen, 87526917@qq.com;
}

Wei Cai, doccaiwei@126.com; Qi-Wei Zang, sqzangqiwei@163.com

This article is available in open access under Creative Common Attribution-Non-Commercial-No Derivatives 4.0 International (CC BY-NC-ND 4.0) license, allowing to download articles and share them with others as long as they credit the authors and the publisher, but without permission to change them in any way or use them commercially. (c) Polish Society for Histochemistry and Cytochemistry 


\section{Introduction}

Lung cancer is the leading cause of cancer-related mortality globally has an estimated 1.4 million deaths each year [1]. Non-small cell lung cancer (NSCLC) accounts for about $70-80 \%$ of all lung cancer types $[2,3]$. Nearly $50 \%$ of patients with stage I NSCLC die within ten years after initial diagnosis [4]. The fiveyear survival rate for patients with NSCLC is merely $15 \%$ due to the clinical complexity $[5,6]$. Therefore, a comprehensive understanding of the molecular mechanisms regarding NSCLC will contribute to the development of diagnostic technologies, new treatment methods, and preventive approaches [7].

Ly-1 antibody reactive clone (LYAR), a $45 \mathrm{kD}$ nucleolar zinc finger protein, was firstly obtained from the T cell leukemia line cDNA library of a mouse [8]. It has been reported that LYAR overexpressing in fibroblast cells has increased the ability to form tumors in nu/nu mice [8]. In addition to its high expression in a variety of leukemia cell lines [8], LYAR was found to be a candidate gene for human neuroblastoma by using comparative genomic hybridization [9]. The previous study has shown that LYAR is a key regulator of cell migration and invasion in colorectal cancer [10]. A gene expression profile study in patients with ovarian cancer revealed that LYAR is a potential prognostic biomarker for ovarian cancer treatment and postoperative supportive therapies [11]. However, the potential function of LYAR in NSCLC is still unclear. Thus, the present study was performed to investigate the clinical significance of LYAR in NSCLC.

\section{Materials and methods}

Patients and tissue samples. One hundred and twenty-one NSCLC samples were collected from patients who underwent salvage ectomy in Nantong Tumor Hospital and The Affiliated Suqian First People's Hospital of Nanjing Medical University from 2016 to 2017. The fresh samples were frozen in liquid nitrogen immediately and maintained at $-80^{\circ} \mathrm{C}$ until Western blot analysis. None of the patients was treated with preoperative therapies. Samples were classified according to the tumor-node-metastasis classification system[12]. This study was approved by the ethics committee of The Affiliated Suqian First People's Hospital of Nanjing Medical University. Written informed consents were obtained from each patient.

Western blot. Proteins were prepared from tumor tissue and cell cultures using RIPA lysis buffer. $25 \mu \mathrm{g}$ total proteins were separated on polyacrylamide gels and then transferred to polyvinylidene fluoride membranes (Millipore, Bedford, MA). The membranes were blocked with $5 \%$ non-fat milk for about $2 \mathrm{~h}$ at room temperature and then were incubat- ed with primary antibodies at $4^{\circ} \mathrm{C}$ overnight. The primary antibodies were as follows: anti-LYAR (Abgent, 1:1000), anti-PCNA (Santa Cruz Biotechnology, 1:1000), anti-GAPDH (Santa Cruz Biotechnology, 1:1000), anti-Cyclin A (Santa Cruz Biotechnology, 1:1000), and anti-Caspase 3 (Santa Cruz Biotechnology, 1:500). Subsequently, the membrane was incubated with corresponding rabbit horseradish peroxidase-linked secondary IgG antibodies (Amersham, Arlington Heights, IL, USA) for $2 \mathrm{~h}$ at room temperature, followed by detection with the enhanced chemiluminescence (Thermo Fisher Scientific, Inc.). The band density was measured by a computer-assisted image-analysis system (Imaging Technology, Ontario, Canada).

Immunohistochemistry. Tumor tissues and adjacent non-tumor tissues samples were fixed in $4 \%$ paraformaldehyde and embedded in the paraffin. Thereafter, tissues were cut into $4 \mu \mathrm{m}$-thick sections. Tissue sections were deparaffinized with xylene, rehydrated with graded ethanol followed by antigen retrieval. After the slices were cooled at room temperature, tissue sections were incubated with LYAR and Ki-67 primary antibodies at $4^{\circ} \mathrm{C}$ overnight and secondary antibodies for $2 \mathrm{~h}$ at room temperature, respectively. Following a final washing in phosphate-buffered saline(PBS), LYAR and Ki-67 signals were visualized with chromogen diaminobenzidine, counterstained with hematoxylin, and mounted in Kayser's glycerine gelatine. For semi-quantitative measurements of LYAR and Ki-67 density, the slides were photographed and analyzed using a computer-aided image-analyzing system (Motic Images Advanced 3.2).

Immunohistochemical evaluation. Immunostained sections were evaluated blinded without knowing the patient's clinical and pathological parameters. For the assessment of LYAR and Ki-67, more than 1000 cells from five high-power fields in each section were chosen randomly. Staining was measured by the percentage of the immunostained cells related to the total number of cells. For the evaluation of the LYAR protein immunoreaction, staining intense was classified as 1 (negatively or poorly staining), 2 (moderately staining), or 3 (strongly staining). Percentage scores were assigned as 1 (negatively or poorly stained), 2 (moderately stained), and 3 (strongly stained). Percentage scores were assigned as 1 (1-25\%), $2(26-50 \%)$ and 3 (51-100\%) for univariate and multivariate analyses. After multiplying the two scores, they were divided into two groups (high expression group and low expression group) according to the scores (average $=4.5$ ) [13].

Cell culture and transient transfection. NSCLC cell line (A549) was purchased from the Cell Bank of Type Culture Collection of the Chinese Academy of Sciences (Shanghai, China). Cells were maintained in Nutrient Mixture F-12 Ham (Sigma-Aldrich, USA) supplemented with $2 \mathrm{mM}$ glutamine, $10 \%$ fetal bovine serum (FBS), and $100 \mathrm{U} / \mathrm{mL}$ 
penicillin-streptomycin mixture (GibCo BRL, Grand Island, $\mathrm{NY}$ ) at $37^{\circ} \mathrm{C}$ and $5 \% \mathrm{CO}_{2}$. Human bronchial epithelial cell line (BEAS-2B) was obtained from the Department of Pathology of Nantong University (Nantong, China) and were cultured in high-glucose DMEM (GibCo BRL, Grand Island, NY, USA) supplemented with $10 \%$ fetal bovine serum, $100 \mathrm{U} / \mathrm{mL}$ penicillin-streptomycin mixture at $37^{\circ} \mathrm{C}$ and $5 \% \mathrm{CO}_{2}$. Lipofectamine ${ }^{\circledast} 2000$ reagent (Invitrogen; Thermo Fisher Scientific, Inc.) was used to perform cell transfection according to the manufacturer's protocol. The knockdown of LYAR in A549 cells was induced using $2 \mu \mathrm{g}$ short hairpin RNA (shRNA, Gene Chem technology) against LYAR, using stable non-specific shRNA $(2 \mu \mathrm{g})$ as the negative control (shRNA-NC). The shRNA-NC sequence was 5'-GAAGCCTAAGAAGCGCAAA-3' and 5'AACAGCGAACCAGTCAATA-3'. The LYAR-shRNA3\# sequence was 5'-AGAACCCTACCTTTAAGTT-3' and 5'-TGGTCATCTTTAACAAGAA-3'. Cells were transfected for $24 \mathrm{~h}$ at $37^{\circ} \mathrm{C}$ in an atmosphere containing $5 \% \mathrm{CO}_{2}$ to obtain stably transfected cells for future use. The knockdown of LYAR was confirmed using Western blotting.

Cell viability assay. According to the manufacturer's introduction, a cell viability assay was carried out using Cell Counting Kit-8 (Dojindo, Kumamoto, Japan). Briefly, cells $\left(2 \times 10^{4}\right.$ cells/well $)$ were seeded in a 96-well plate with $100 \mu \mathrm{L}$ culture medium and incubated in a $\mathrm{CO}_{2}$ incubator at $37^{\circ} \mathrm{C}$ overnight. Then, the Counting Kit-8 reagent (Dojindo, Japan) was added and cells were incubated at $37^{\circ} \mathrm{C}$ for $2 \mathrm{~h}$. Finally, absorbance was recorded at $450 \mathrm{~nm}$ using a microplate reader (BioRad, Hercules, CA, USA).

Flow cytometry. For cell cycle analysis, A549 cells were seeded at a density of $2.5 \times 10^{5}$ cells per well in 24 -well plates. After incubation for $24 \mathrm{~h}$, the cells were collected and fixed with cold ethanol $\left(70 \% \mathrm{v} / \mathrm{v},-20^{\circ} \mathrm{C}, 30 \mathrm{~min}\right)$. Then, cells were incubated with RNase $\mathrm{A}\left(1 \mathrm{mg} / \mathrm{mL}, 30 \mathrm{~min}\right.$ at $\left.37^{\circ} \mathrm{C}\right)$. Subsequently, cells were stained with $50 \mu \mathrm{g} / \mathrm{mL}$ propidium iodide and analyzed using a Becton-Dickinson flow cytometer BD FACScan (CA, USA).

Terminal-deoxynucleotidyl-transferase-mediated dUTP-biotin nick end labeling (TUNEL) assay. TUNEL assay was performed as described previously [14]. A549 cells were seeded at a density of $2.5 \times 10^{5}$ cells per well in 24-well plates and incubated for $24 \mathrm{~h}$. Then, cells were transfected with shRNA-LYAR or shRNA-NC for another $48 \mathrm{~h}$. Subsequently, cells were subjected to TUNEL staining by using an In Situ Cell Death Detection Kit (Roche, Mannheim, Germany). Cells were fixed in paraformaldehyde $\left(4 \%, 4^{\circ} \mathrm{C}\right.$ for $\left.30 \mathrm{~min}\right)$, and then treated with $0.1 \%$ TritonX-100, labeled with fluorescein-12-dUTP using terminal deoxynucleotidyl transferase. Images were

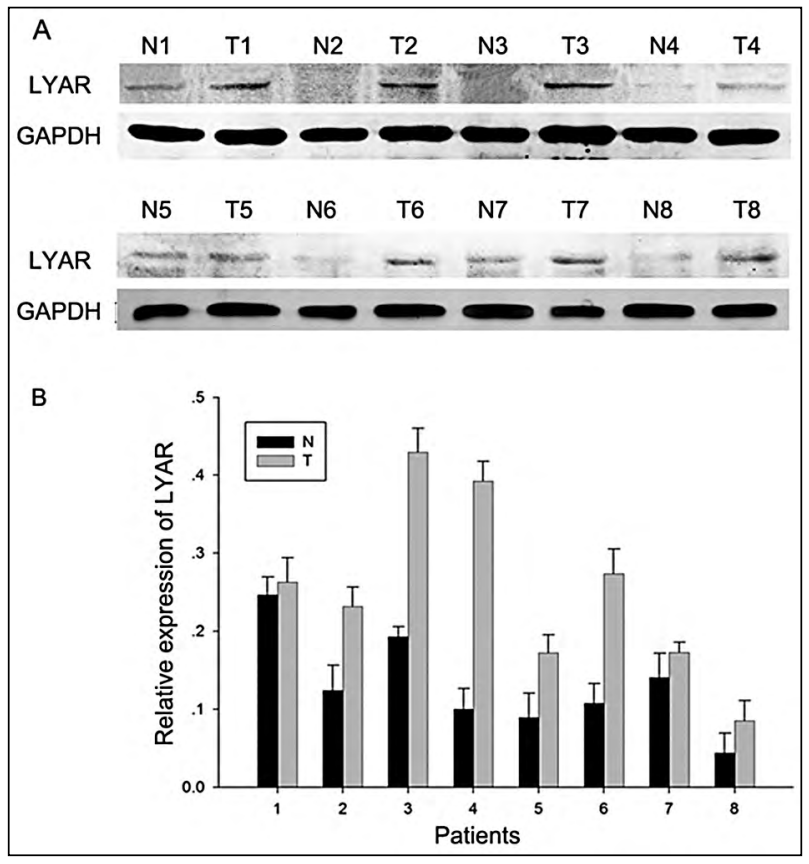

Figure 1. LYAR protein expression was increased in NSCLC tissues. A. Western blot was performed to determine the expression of LYAR in eight representatives paired NSCLC tumor tissues (T) and adjacent non-tumor tissues (N). B. Quantification of the expression of LYAR in eight representatives paired NSCLC tumor tissues (T) and adjacent non-tumor tissues $(\mathrm{N})$. NSCLC, non-small cell lung cancer; LYAR, Ly-1 antibody reactive clone.

detected by fluorescence microscopy (Leica, DM 5000B; LeicaCTR 5000; Wetzlar, Germany).

Statistical analysis. SPSS version 13.0 (SPSS Institute. IL, USA) was used for statistical analyses. Quantitative data were expressed as means \pm standard deviations $(\mathrm{SD})$ and compared by Student's $t$-test. Qualitative data were expressed as numbers and percentages, and were compared using the $\chi^{2}$ test. Kaplan-Meier method was used to calculate the survival curve. Spearman's correlation test was used for the correlation analysis among the LYAR and Ki-67 expression. Cox's proportional hazards model was used to perform multivariate analysis. Statistical significance was set at $p<0.05$.

\section{Results}

\section{LYAR expression was increased in NSCLC tissues}

To explore the relationship between LYAR and NSCLC, we first examined the expression of LYAR at protein levels at eight NSCLC tissues and their adjacent noncancerous tissues by Western blot. As shown in Figure 1, the expression of LYAR was dramatically 


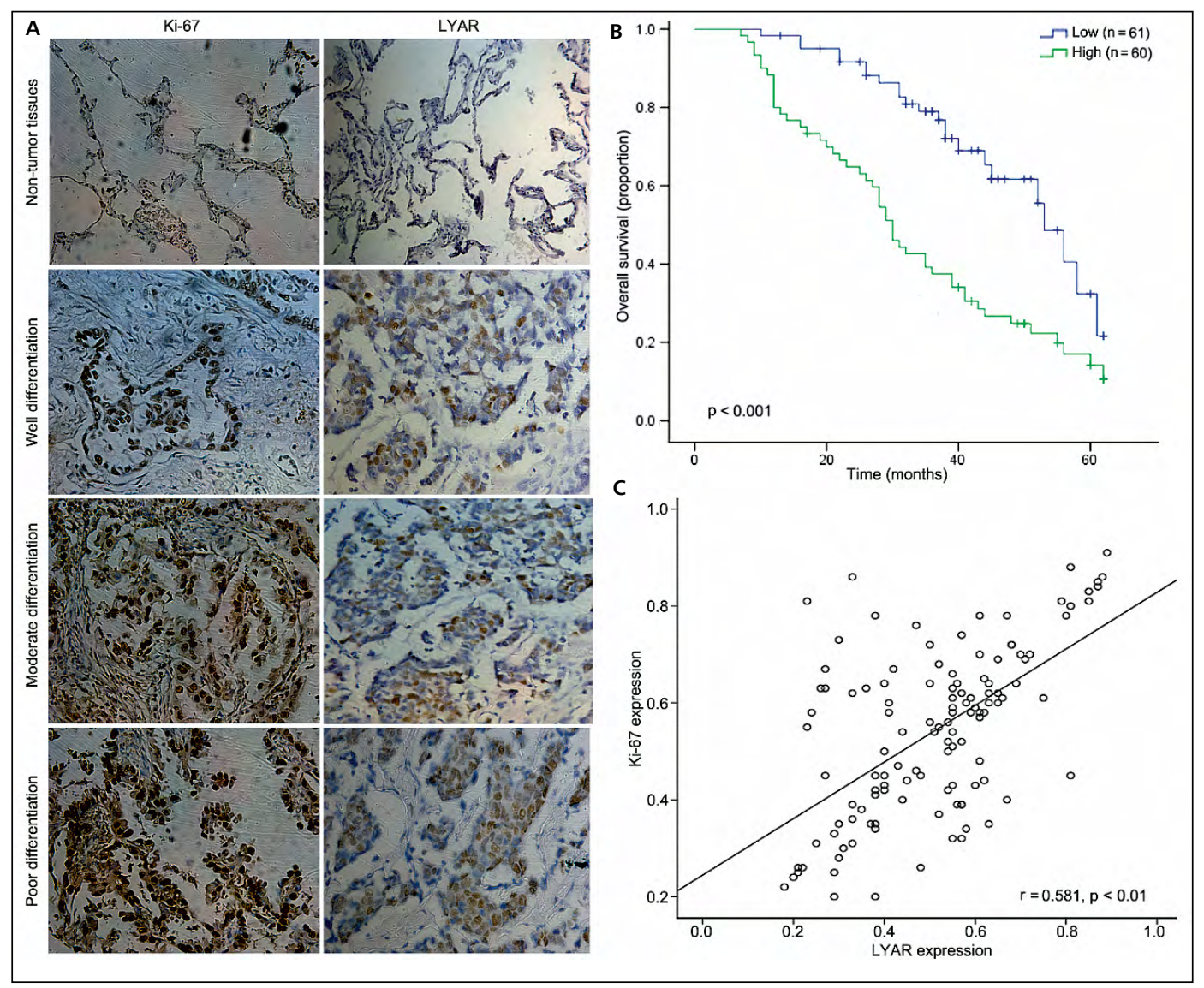

Figure 2. LYAR immunoreactivity was correlated with Ki-67 and NSCLC patients' survival. A. Representative images of immunohistochemical staining of LYAR and Ki-67 in 121 NSCLC tissues and adjacent non-tumor tissues $(200 \times)$. B. LYAR protein overexpression predicts a poor prognosis for NSCLC patients. C. Correlation analysis between LYAR and Ki-67 expression. Data were presented as mean \pm SD. NSCLC, non-small cell lung cancer; LYAR, Ly-1 antibody reactive clone.

increased in NSCLC tissues compared with normal noncancerous tissues.

\section{LYAR expression was correlated with Ki-67 and NSCLC patients' survival}

To explore the clinic significance of LYAR in NSCLC, 121 NSCLC samples and their adjacent noncancerous tissues were selected. The expression of LYAR and Ki-67 (a cell proliferation-associated nuclear marker) was determined by immunohistochemistry (Fig. 2A). Immunoreactivity of both LYAR and Ki-67 were seen predominantly in the nucleus. As expected, the expression of LYAR and Ki67 were up-regulated in NSCLC tissues compared with their adjacent noncancerous tissues. In addition, the expression of LYAR in poorly differentiated samples was higher than that in well-differentiated samples, which was consistent with Ki-67.

The histopathological and clinical characteristics of the 121 NSCLC patients were shown in Table 1. In consistence with the results shown in Figure 2A, the expression of LYAR was related to the clinical stage, histological differentiation, and Ki-67 expression (all $p<0.05$, Table 1). In addition, Kaplan-Meier analysis showed that the overall survival rate in patients with LYAR high expression was significantly lower than that with LYAR low expression ( $p<0.05$, Fig. 2B). Cox's proportional hazard regression model showed that LYAR expression, tumor size, histological differentiation, and Ki-67 expression were independent prognostic factors for overall survival (Table 2).

In order to further confirm the correlation between LYAR and Ki-67 expression, Spearman's correlation test was performed by a percentage of positive malignant cells. As shown in Figure 2C, a positive correlation was found between LYAR and Ki-67 expression $(\mathrm{r}=0.581, p<0.01)$. Taken together, these results indicated that LYAR could predict poor prognosis of NSCLC, and may be involved in the proliferation of NSCLC cells. 
Table 1. The characteristics and histopathological data of patients

\begin{tabular}{|c|c|c|c|c|}
\hline \multirow[t]{2}{*}{ Characteristic } & \multirow[t]{2}{*}{ Total } & \multicolumn{2}{|c|}{ LYAR expression } & \multirow[t]{2}{*}{$p$-value } \\
\hline & & Low & High & \\
\hline Age (year, n/\%) & & & & 0.935 \\
\hline$<60$ & $54(44.63)$ & $27(22.31)$ & $27(22.31)$ & \\
\hline$\geq 60$ & $67(55.37)$ & $34(28.10)$ & $33(27.27)$ & \\
\hline Gender (n, \%) & & & & 0.085 \\
\hline Male & $64(52.89)$ & $37(30.58)$ & $27(22.31)$ & \\
\hline Female & $57(47.11)$ & $24(19.84)$ & $33(27.27)$ & \\
\hline Smoking $(\mathrm{n}, \%)$ & $20(16.53)$ & $1(0.83)$ & $19(15.70)$ & 0.150 \\
\hline Clinical stage (n, \%) & & & & 0.007 \\
\hline I & $77(63.64)$ & $47(38.84)$ & $30(24.79)$ & \\
\hline II & $28(23.14)$ & $10(8.26)$ & $18(14.88)$ & \\
\hline III & $16(13.22)$ & $4(3.31)$ & $12(9.92)$ & \\
\hline Tumor size (n, \% ) & & & & 0.000 \\
\hline$<3 \mathrm{~cm}$ & $52(42.98)$ & $36(29.75)$ & $16(13.22)$ & \\
\hline$\geq 3 \mathrm{~cm}$ & $69(57.02)$ & $25(20.66)$ & $44(36.36)$ & \\
\hline Differentiation (n, \%) & & & & 0.001 \\
\hline Well & $14(11.57)$ & $1(0.83)$ & $13(10.74)$ & \\
\hline Moderate & $81(66.94)$ & $43(35.54)$ & $38(31.40)$ & \\
\hline Poor & $26(21.49)$ & $17(14.05)$ & $9(7.44)$ & \\
\hline Lymph node status (n, \%) & & & & 0.003 \\
\hline 0 & $80(66.12)$ & $48(39.67)$ & $32(26.45)$ & \\
\hline$>0$ & $41(33.88)$ & $13(10.74)$ & $28(23.14)$ & \\
\hline Ki-67 expression (n, \%) & & & & 0.000 \\
\hline Low & $52(42.98)$ & $38(31.40)$ & $14(11.57)$ & \\
\hline High & $69(57.02)$ & $23(19.01)$ & $46(38.02)$ & \\
\hline
\end{tabular}

LYAR — Ly-1 antibody reactive clone

Table 2. Contribution of various potential prognostic factors to survival by Cox regression analysis in 121 NSCLC specimens

\begin{tabular}{|l|c|c|c|}
\hline Parameters & Hazard ratio & 95\% confidence interval & $p$-value \\
\hline Age & 1.037 & $0.632-1.701$ & 0.886 \\
\hline Gender & 1.700 & $1.004-2.879$ & 0.480 \\
\hline Tumor size & 3.600 & $1.524-8.503$ & 0.003 \\
\hline Clinical stage & 1.513 & $0.905-2.528$ & 0.114 \\
\hline Lymph node metastasis & 1.475 & $0.851-2.555$ & 0.166 \\
\hline Pathology grade & 0.485 & $0.280-0.839$ & 0.010 \\
\hline LYAR expression & 1.931 & $1.084-3.439$ & 0.026 \\
\hline Ki-67 expression & 0.382 & $0.161-0.906$ & 0.029 \\
\hline
\end{tabular}

LYAR — Ly-1 antibody reactive clone

\section{LYAR affected the cell cycle progression of NSCLC cells}

Considering the correlation between LYAR and Ki-67 expression, we hypothesized that LYAR may be involved in the cell cycle progression of NSCLC cells. A549 cells were cultured in a serum-free conditioned medium for $72 \mathrm{~h}$ and then recovered by adding a medium containing $10 \%$ FBS. Following that, a Western blot was performed to evaluate the expression of LYAR, along with PCNA (a marker 


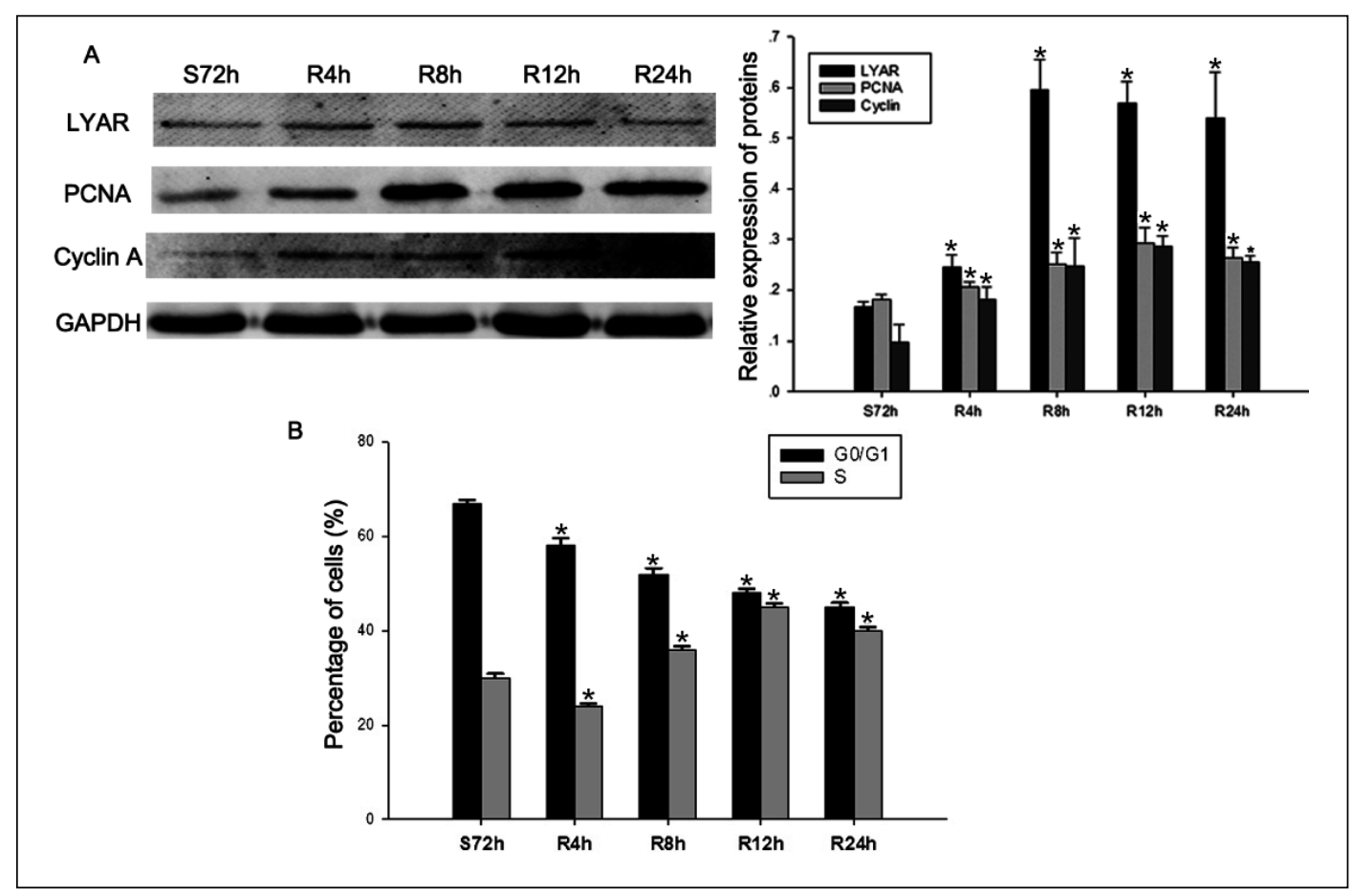

Figure 3. LYAR affected the cell cycle progression of NSCLC cells. A. Cells were synchronized at G0/G1 after serum starvation for $72 \mathrm{~h}(\mathrm{~S} 72 \mathrm{~h})$, then progressed into the cell cycle by adding a medium containing $10 \%$ FBS for the indicated times (R4h, R8h, R12h, R24h). The expression of LYAR, PCNA, and cyclin A was analyzed by Western blot. B. Cell cycle distribution of A549 cells was analyzed by flow cytometry assay. Data were presented as mean \pm SD. NSCLC, non-small cell lung cancer; LYAR, Ly-1 antibody reactive clone; S, serum starvation; R, serum re-addition; * $p<0.05$, compared with S72h.

of proliferation) and Cyclin A (a marker of the cell cycle) during cell cycle progression. As expected, the expression of LYAR significantly increased upon serum re-addition after serum starvation for $72 \mathrm{~h}$, with a concomitant up-regulation of PCNA and Cyclin A (all $p<0.05$, Fig. 3A).

Furthermore, a flow cytometric assay was used to analyze the cell cycle progression after serum deprivation and re-addition. The results showed that A549 cells were arrested in the G1 phase after serum deprivation for $72 \mathrm{~h}$ (Fig. 3B). After serum re-addition for $24 \mathrm{~h}$, the number of A549 cells at the $\mathrm{S}$ phase increased gradually from $28.11 \%$ to $40.08 \%(p<0.05)$. The above results indicated that LYAR might have an impact on the cell cycle progression of NSCLC cells.

\section{LYAR promoted the proliferation and inhibited the apoptosis of NSCLC cells}

To further confirm the role of LYAR in the proliferation of NSCLC cells, A549 cells were selected to construct LYAR knocked-down cells. The efficiency of transfection was confirmed by Western blot. As shown in Figure 4A, LYAR-shRNA\#3 transfected A549 cells showed the most significant decrease in LYAR expression $(\mathrm{p}<0.05)$. Therefore, the LYAR-shR-
NA\#3 was used for the following experiments. Western blot showed that the knockdown of LYAR with shRNA\#3 dramatically decreased the expression of PCNA and increased cleaved-Caspase3, compared with the shRNA-NC (Fig. 4B). CCK-8 assays were used to determine the effect of LYAR on cell proliferation. As shown in Fig. 4C, the proliferative ability of A549 cells transfected with LYAR-shRNA\#3 was significantly inhibited compared with cells transfected with shRNA-NC $(p<0.05)$. Furthermore, flow cytometric assay of A549 cells which were transfected with LYAR-shRNA\#3 showed the number of cells at the G0/G1 phase increased from $52.61 \%$ to $64.95 \%$, and the number of cells at the S phase decreased from $31.89 \%$ to $26.44 \%$ (Fig. 4D). TUNEL assay was used to explore the effect of LYAR on cell apoptosis. The result showed that the knockdown of LYAR induced the apoptosis of A549 cells (Fig. 4E). These results indicated that LYAR may promote the proliferation and inhibit the apoptosis of NSCLC cells.

\section{Discussion}

The 5-year survival rate of patients with NSCLC is still very low (about 15\%), despite the advances in 


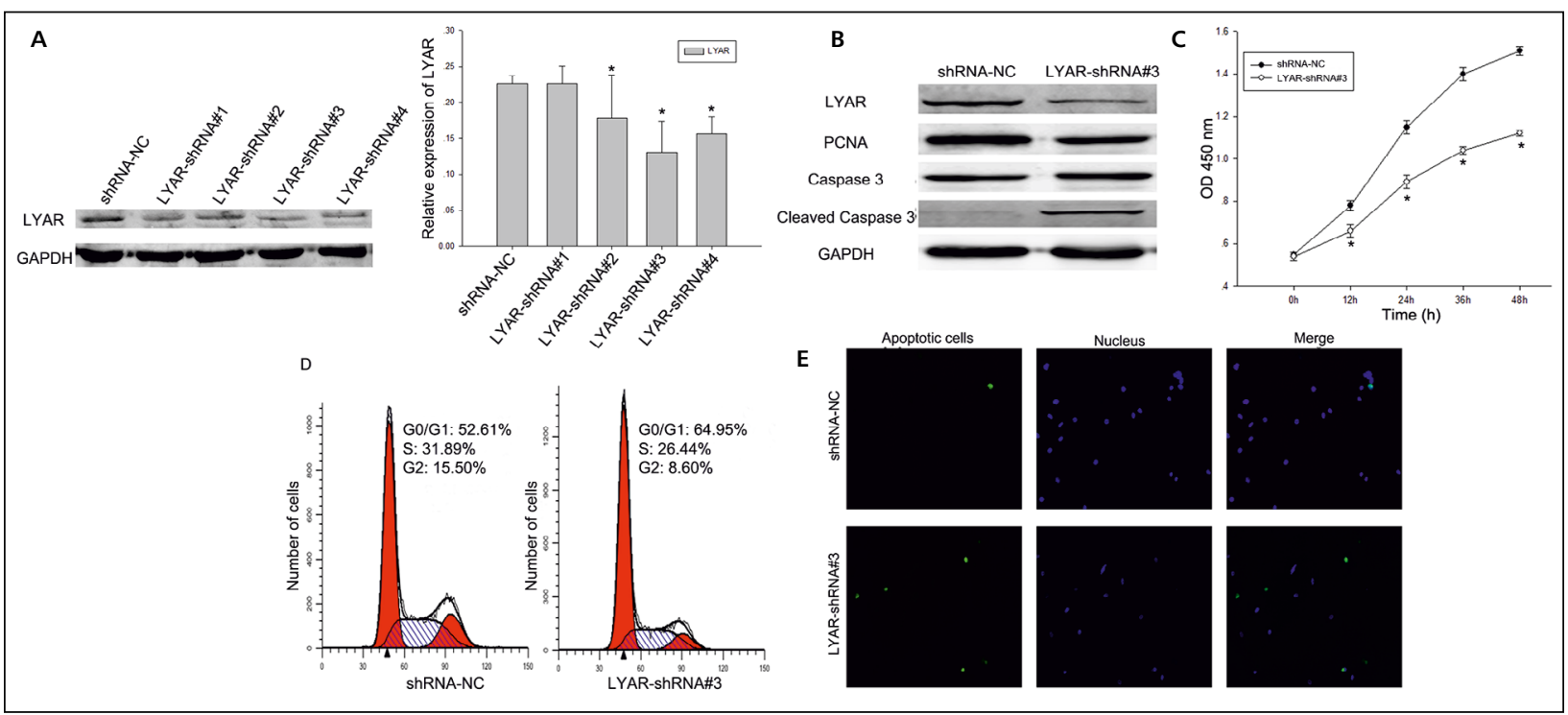

Figure 4. LYAR promoted the proliferation and inhibited the apoptosis of NSCLC cells. A. Expression levels of LYAR in A549 cells transfected with shRNA-NC or LYAR-shRNA were determined using Western blot. B. Western blot analysis of LYAR, PCNA, Caspase 3, and cleaved-Caspase 3 expressions in A549 cells transfected with shRNA-NC or LYAR-shRNA\#3. C. Cell proliferation was measured using a Cell Counting Kit-8 assay in A549 cells transfected with shRNA-NC or LYAR-shRNA\#3. D. Cell cycle distribution was analyzed by flow cytometric assay in A549 cells transfected with shRNA-NC or LYAR-shRNA\#3. E. Cell apoptosis was analyzed using a TUNEL assay in A549 cells transfected with shRNA-NC or LYAR-shRNA\#3. Data were presented as mean \pm SD. NSCLC, non-small cell lung cancer; LYAR, Ly-1 antibody reactive clone; NC, negative control; shRNA, short hairpin RNA; " $p<0.05$, compared with shRNA-NC.

the treatment of NSCLC recently [5, 6]. Therefore, detailed NSCLC progression researches are necessary for improving the prognosis of NSCLC. LYAR is a nuclear protein that consists of 379 amino acids with three copies of nuclear localization signals and a zinc-finger motif [15]. Evidence has shown that LYAR can increase the tumorigenicity of the fibroblast cells in nu/nu mice, and may function as a novel nucleolar oncoprotein in regulating cell growth [8]. In addition, several reports have shown that LYAR is involved in various types of cancer, such as colorectal cancer [10], ovarian cancer [11], and leukemia [8]. However, to the best of our knowledge, the role of LYAR in lung cancer is still unknown. The present study aimed to investigate the clinical significance of LYAR in NSCLC.

In the current study, we found that the expression level of LYAR in NSCLC tissues was higher than that in their adjacent noncancerous tissues. Kaplan-Meier analysis revealed that the high expression of LYAR predicted poor survival of NSCLC. These results were consistent with the report by Sun et al., which demonstrated that high levels of LYAR expression in human neuroblastoma tissues predicted poor event-free and overall survival of patients with neuroblastoma [16]. The present study also showed that the LYAR expression showed a positive correlation with the Ki-67 expression. Ki-67 was a nuclear protein expressed in the M, G1, S, and G2 phases of the cell cycle, but not in the G0 phase [17]. Thus, Ki-67 was usually used as a marker for the proliferation of various tumor cells [18]. Interestingly, evidence had shown that the LYAR gene was not expressed during the proliferation of some normal cells [8], suggesting that LYAR was associated with abnormal cell growth. Taken together, our results revealed that LYAR may be involved in the proliferation of NSCLC cells.

To further confirm the role of LYAR in cell proliferation, a series of in vitro experiments were designed. Serum starvation and re-addition experiments showed that LYAR expression was associated with cell proliferation, and LYAR knockdown in A549 cells inhibited the cell proliferation and induced the G0/G1 cell cycle arrest. Similarly, LYAR overexpression was found to promote the proliferation of NIH-3T3 cells (a fibroblast-like line derived from a murine embryo) [19]. These results indicated that LYAR may promote the proliferation of NSCLC cells. One possible mechanism for the role of LYAR in cell proliferation was maintaining the homeostasis level of nucleolin, which is a multifunctional RNA-binding protein [20]. It has been reported that LYAR could form a complex with nucleolin and prevent its self-cleavage to promote cell proliferation [21]. However, the mechanism linking 
LYAR-mediated increase in cell proliferation needs to be determined in further investigations.

In conclusion, the present study revealed the clinical significance of LYAR in NSCLC. The results showed that LYAR was highly expressed in NSCLC tissues and closely correlated to the survival of patients with NSCLC. Additionally, a significantly positive correlation was found between LYAR and Ki67 expressions. Finally, LYAR knockdown inhibited the proliferation and induced the G0/G1 cell cycle arrest and apoptosis of NSCLC cells. Taken together, our study demonstrated that LYAR might serve as a tumor promoter in NSCLC progression by promoting the proliferation and inhibiting the apoptosis of NSCLC cells. Inhibiting the expression of LYAR was considered as a potential novel therapeutic strategy for NSCLC.

\section{Funding}

This work was supported by the Science and Technology Project of Nantong (number MSZ18215) and the Young Science and Technology Talents Project of Natural Science Foundation (number k202008).

\section{Author contributions}

Conception and design: Q-W Zang, X-N Lu, and G-J $\mathrm{Ju}$; Administrative support: Q-W Zang; Materials and samples provided: Y-X Wang and Y-L Wang; Data collection and collation: K Wang and J-L Chen; Data analysis and interpretation: W Cai; Drafting article, X-N Lu and G-J Ju. All the authors have read and approved the final manuscript.

\section{Data availability statements}

The data underlying this article are available in the article.

\section{Experimental ethics}

This study was approved by the ethics committee of The Affiliated Suqian First People's Hospital of Nanjing Medical University.

\section{Conflict of interests}

The authors declare that they have no conflict of interest.

\section{References}

1. Siegel R, Naishadham D, Jemal A. Cancer statistics, 2012. CA Cancer J Clin. 2012; 62(1): 10-29, doi: 10.3322/caac.20138, indexed in Pubmed: 22237781.
2. Yang Z, He J, Gao P, et al. miR-769-5p suppressed cell proliferation, migration and invasion by targeting TGFBR1 in non-small cell lung carcinoma. Oncotarget. 2017; 8(69): 113558-113570, doi: 10.18632/oncotarget.23060, indexed in Pubmed: 29371929.

3. Xu XL, Gong Y, Zhao DP. Elevated PHD2 expression might serve as a valuable biomarker of poor prognosis in lung adenocarcinoma, but no lung squamous cell carcinoma. Eur Rev Med Pharmacol Sci. 2018; 22(24): 8731-8739, doi: 10.26355/ eurrev_201812_16638, indexed in Pubmed: 30575913.

4. Ou SHI, Zell JA, Ziogas A, et al. Prognostic factors for survival of stage I nonsmall cell lung cancer patients : a population-based analysis of 19,702 stage I patients in the California Cancer Registry from 1989 to 2003. Cancer. 2007; 110(7): 1532-1541, doi: 10.1002/cncr.22938, indexed in Pubmed: 17702091.

5. Cai J, Fang L, Huang Y, et al. miR-205 targets PTEN and PHLPP2 to augment AKT signaling and drive malignant phenotypes in non-small cell lung cancer. Cancer Res. 2013; 73(17): 5402-5415, doi: 10.1158/0008-5472.CAN-13-0297, indexed in Pubmed: 23856247.

6. Torre LA, Bray F, Siegel RL, et al. Global cancer statistics, 2012. CA Cancer J Clin. 2015; 65(2): 87-108, doi: 10.3322/ caac.21262, indexed in Pubmed: 25651787.

7. Kim IM, Ackerson T, Ramakrishna S, et al. The Forkhead Box $\mathrm{m} 1$ transcription factor stimulates the proliferation of tumor cells during development of lung cancer. Cancer Res. 2006; 66(4): 2153-2161, doi: 10.1158/0008-5472.CAN-05-3003, indexed in Pubmed: 16489016.

8. Su L, Hershberger RJ, Weissman IL. LYAR, a novel nucleolar protein with zinc finger DNA-binding motifs, is involved in cell growth regulation. Genes Dev. 1993; 7(5): 735-748, doi: 10.1101/gad.7.5.735, indexed in Pubmed: 8491376.

9. Swartling FJ, Grimmer MR, Hackett CS, et al. Pleiotropic role for MYCN in medulloblastoma. Genes Dev. 2010; 24(10): 1059-1072, doi: 10.1101/gad.1907510, indexed in Pubmed: 20478998.

10. Wu Y, Liu M, Li Z, et al. LYAR promotes colorectal cancer cell mobility by activating galectin-1 expression. Oncotarget. 2015; 6(32): 32890-32901, doi: 10.18632/oncotarget.5335, indexed in Pubmed: 26413750.

11. Isaksson HS, Sorbe B, Nilsson TK. Whole genome expression profiling of blood cells in ovarian cancer patients -prognostic impact of the CYP1B1, MTSS1, NCALD, and NOP14. Oncotarget. 2014; 5(12): 4040-4049, doi: 10.18632/oncotarget.1938, indexed in Pubmed: 24961659.

12. Kerr KM. Clinical relevance of the new IASLC/ERS/ATS adenocarcinoma classification. J Clin Pathol. 2013; 66(10): 832-838, doi: 10.1136/jclinpath-2013-201519, indexed in Pubmed: 23564952.

13. Lv L, Wan C, Chen B, et al. Nemo-like kinase (NLK) inhibits the progression of NSCLC via negatively modulating WNT signaling pathway. J Cell Biochem. 2014; 115(1): 81-92, doi: 10.1002/jcb.24635, indexed in Pubmed: 23904219.

14. Xue Q, Lv L, Wan C, et al. Expression and clinical role of small glutamine-rich tetratricopeptide repeat (TPR)-containing protein alpha (SGTA) as a novel cell cycle protein in NSCLC. J Cancer Res Clin Oncol. 2013; 139(9): 1539-1549, doi: 10.1007/s00432-013-1474-5, indexed in Pubmed: 23857189.

15. Miyazawa N, Yoshikawa $\mathrm{H}$, Magae S, et al. Human cell growth regulator Ly-1 antibody reactive homologue accelerates processing of preribosomal RNA. Genes Cells. 2014; 19(4): $273-$ -286, doi: 10.1111/gtc.12129, indexed in Pubmed: 24495227.

16. Sun Y, Atmadibrata B, Yu D, et al. Upregulation of LYAR induces neuroblastoma cell proliferation and survival. 
Cell Death Differ. 2017; 24(9): 1645-1654, doi: 10.1038/ cdd.2017.98, indexed in Pubmed: 28686580.

17. Ishibashi N, Maebayashi T, Aizawa T, et al. Correlation between the Ki-67 proliferation index and response to radiation therapy in small cell lung cancer. Radiat Oncol. 2017; 12(1): 16, doi: 10.1186/s13014-016-0744-1, indexed in Pubmed: 28086989.

18. Zhao WP, Wang HW, Liu J, et al. Positive PCNA and Ki67 Expression in the Testis Correlates with Spermatogenesis Dysfunction in Fluoride-Treated Rats. Biol Trace Elem Res. 2018; 186(2): 489-497, doi: 10.1007/s12011-018-1338-6, indexed in Pubmed: 29748930.
19. Yonezawa K, Sugihara Y, Oshima K, et al. Lyar, a cell growth-regulating zinc finger protein, was identified to be associated with cytoplasmic ribosomes in male germ and cancer cells. Mol Cell Biochem. 2014; 395(1-2): 221-229, doi: 10.1007/s11010-014-2128-x, indexed in Pubmed: 24990247.

20. Abdelmohsen K, Gorospe M. RNA-binding protein nucleolin in disease. RNA Biol. 2012; 9(6): 799-808, doi: 10.4161/ rna.19718, indexed in Pubmed: 22617883.

21. Li H, Wang B, Yang A, et al. Ly-1 antibody reactive clone is an important nucleolar protein for control of self-renewal and differentiation in embryonic stem cells. Stem Cells. 2009; 27(6): 1244-1254, doi: 10.1002/stem.55, indexed in Pubmed: 19489080.

Submitted: 9 April, 2021

Accepted after reviews: 2 December, 2021 Available as AoP: 10 December, 2021 